\title{
Microstructural Characterization and Mechanical Properties of PA11 Nanocomposite Fibers
}

Paulina Latko, Dorota Kolbuk, Rafal Kozera, and Anna Boczkowska

\author{
(Submitted July 21, 2015; in revised form November 24, 2015; published online December 18, 2015)
}

\begin{abstract}
Polyamide 11/multi-walled carbon nanotubes nanocomposite fibers with weight fraction 2, 4, and 6 wt.\% and diameter $80 \mu \mathrm{m}$ were prepared with a twin screw mini-extruder. The morphology and degree of dispersion of the multi-walled carbon nanotubes in the fibers was investigated by using scanning and transmission electron microscopy. In turn, the molecular structure was indicated by using wide-angle $x$-ray scattering and correlated with thermal analysis. It was found that carbon nanotubes lead to the formation of $\alpha$ phase in the fibers and they show medial level of alignment within the length of the fiber. Mechanical analysis of the fibers shows that apart from the crystallinity content, the tensile strength is strongly dependent on the macroscopic defects of the surface of the fibers. Nanocomposite fibers based on polyamide 11 with carbon nanotubes can be used as a precursor for non-woven or woven fabrics manufacturing process.
\end{abstract}

Keywords aerospace, electron microscopy, nanomaterials, thermal analysis, $\mathrm{x}$-ray

\section{Introduction}

Polyamides (nylons) are wide range groups of polymers that are both crystalline and amorphous materials in which the repetitive units are connected by characteristic amide groups (-CONH-). Within this group of polymers, Polyamide 11 (PA11) is one of great importance because of its excellent properties, including resistance to chemicals, a wide range of working temperatures (from -40 to $+130{ }^{\circ} \mathrm{C}$ ), less water absorption or high dimensional stability. Hence, this type of polyamide is willingly used in almost each industrial sectors, for instance, automotive, industrial vehicles, medicine, food packaging, aerospace, sports applications, and also textile industries. The last one includes usage of PA11 fibers as precursors for woven or non-woven fabrics or mats. Nowadays, a lot of researches are focused on electrically conductive fibers and fabrics which can be used as smart materials to regulate electrostatic discharge (ESD) and determine shielding from electromagnetic interference (EMI) and radio frequency interference (RFI). One of the methods to manufacture smart fibers and fabrics is doping neat polymer with conductive filler like multi-walled carbon nanotubes (MWCT, MWCTs). By addition

Paulina Latko, Rafal Kozera, and Anna Boczkowska, Faculty of Materials Science and Engineering, Warsaw University of Technology, ul. Woloska 141, 02-507 Warsaw, Poland; and Technology Partners Foundation, ul. Pawinskiego 5A, 02-106 Warsaw, Poland; and Dorota Kolbuk, Institute of Fundamental Technological Research, Polish Academy of Sciences, ul. Pawinskiego 5B, 02-106 Warsaw, Poland. Contact e-mails: Paulina.Latko@inmat.pw.edu.pl and rafal.kozera@ inmat.pw.edu.pl. of MWCT into a polymer the fibers with improved mechanical performance and electrical conductivity can be achieved (Ref 1-7).

Polymer/CNT nanocomposite fibers with CNT content not higher than $10 \mathrm{wt} . \%$ can be obtained by extrusion or spinning methods. In many cases, the higher the percentage of CNT (more than 10 wt.\%), the lower the desired properties. Nevertheless, the enhancement in mechanical properties, chemical resistance, and increase in electrical and thermal conductivities caused by CNT addition were observed. Moreover, the addition of CNT leads to polymer crystallization, which acted as a template for polymer chain orientation (Ref 4-8).

It has been found that PA11 can occur in several crystal structures that have been described by many authors (Ref 9-18), and hence, some difference in nomenclature is evident. At room temperature (after cooling from the melt), PA11 occurs in the triclinic $\alpha$ form (stable) and smectic pseudo-hexagonal $\delta^{\prime}$-form (metastable). However, the $\alpha$-form in the range of $60-100{ }^{\circ} \mathrm{C}$ alters reversibly into the hexagonal $\delta$ phase, which is stable above $100{ }^{\circ} \mathrm{C}$ (so called Brill transition). According to different authors (Ref 12-17), the specific order of the $\alpha$-form can be reached by slow cooling, annealing, or by solution casting. In turn, a disordered pseudo-hexagonal $\delta^{\prime}$-form can be obtained upon quenching the polymer from the melt state resulting in smectic-like order. In our case, the lattice parameters of the triclinic $\alpha$ crystalline phase are $a=4.9 \AA$, $b=5.4 \AA \AA, c=14.9 \AA \dot{\alpha}, \alpha=49^{\circ}, \beta=77^{\circ}, \gamma=63^{\circ}(\operatorname{Ref} 16)$.

Based on our knowledge, there are just a few papers that investigate nanocomposites made of PA11 with MWCT (Ref 19-22) but not in the form of fibers. We have already published the general procedure of fabrication PA11 fibers and non-woven fabrics doped with MWCT (Ref 23). In this paper, we focused on characterization of PA11 nanocomposite fibers (PA11/MWCT) with 2, 4, and 6 wt.\% of MWCT produced by the drawing of extrudate with average thickness $80 \mu \mathrm{m}$. The effect of MWCT on morphology, their distribution, and PA11 crystallization were evaluated using SEM, TEM, WAXS, and DSC methods. The mechanical properties of PA11/MWCT fibers are shown in terms of further applications. 


\section{Experimental}

\subsection{Materials}

Nanocomposite fibers made of PA11 (Rilsan, Arkema) with a 2 wt.\% MWCT (PA11_2), 4 wt.\% MWCT (PA11_4), and
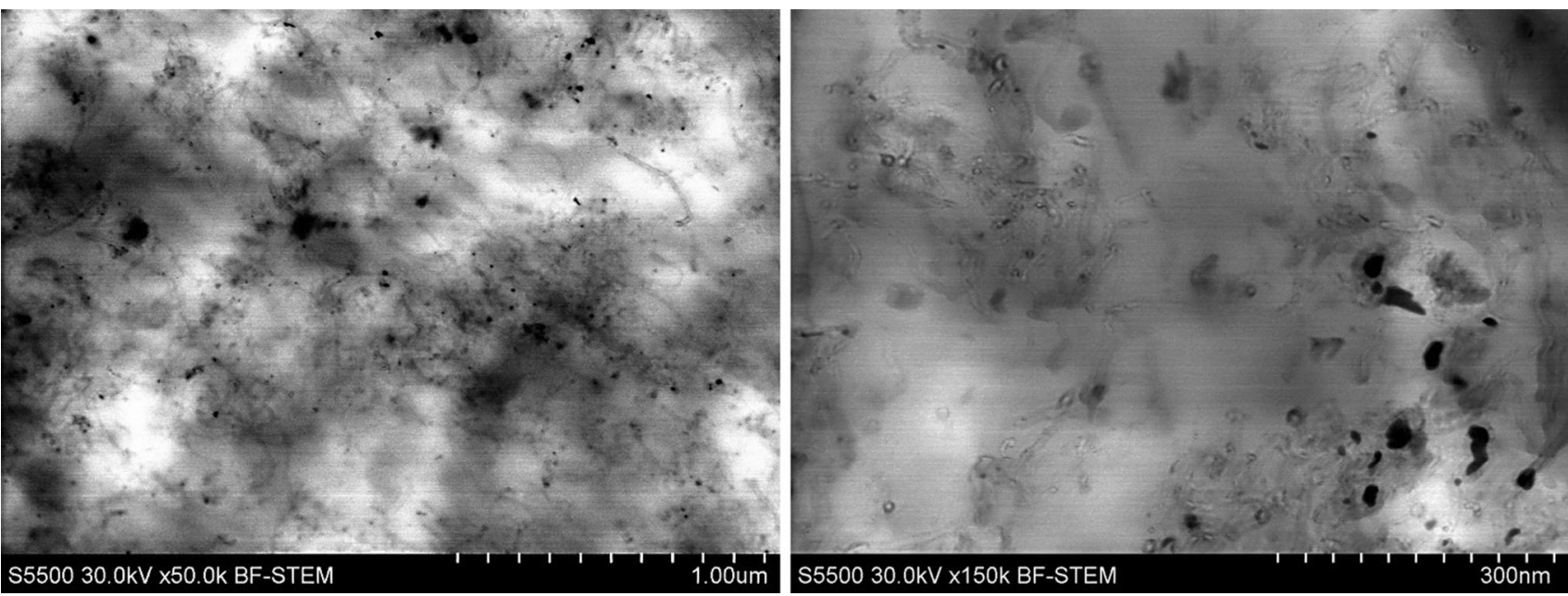

Fig. 1 TEM images of PA11_6 starting pellets
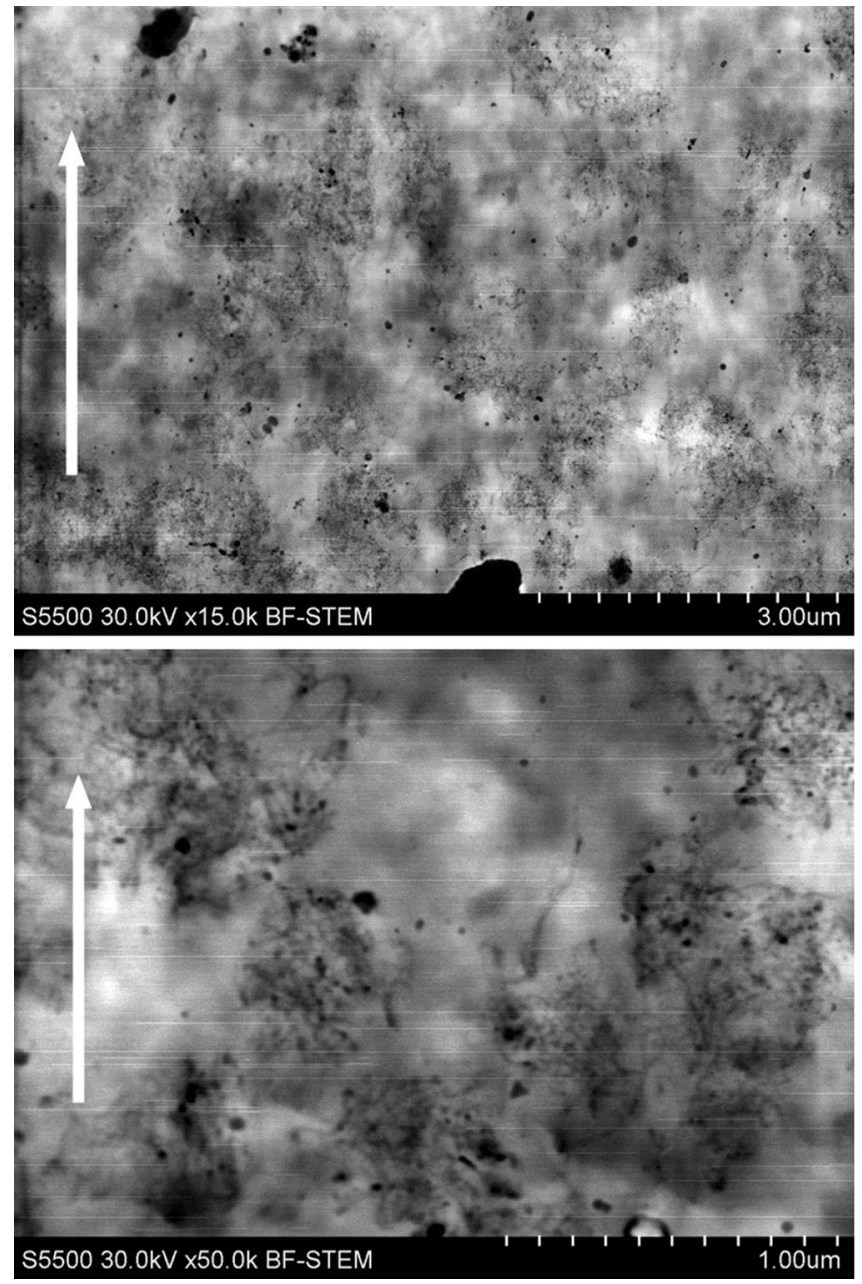

\section{(1)}

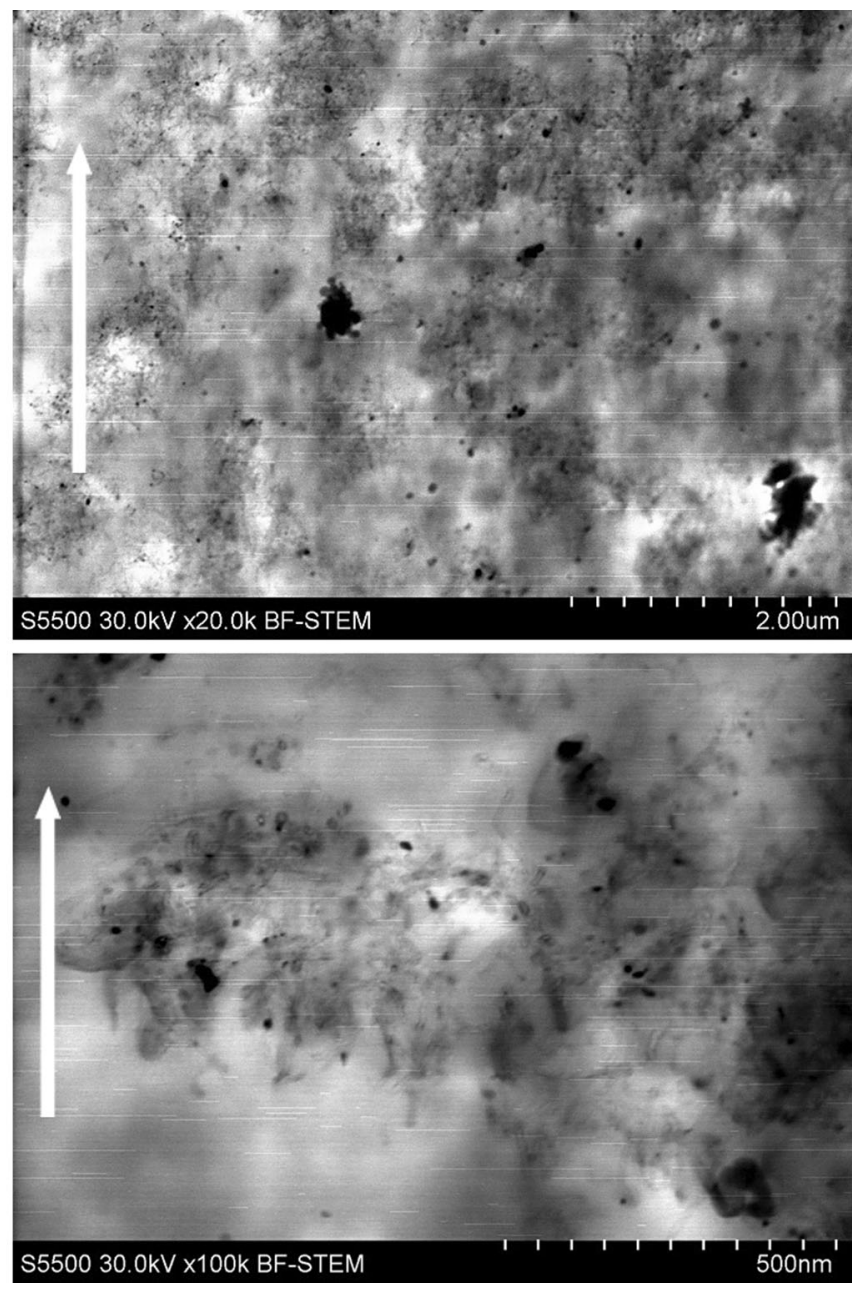

6 wt.\% MWCT (PA11_6) were fabricated using a twin, corotating screw mini-extruder HAAKE MiniLab (Thermo Scientific, Germany) equipped with circular nozzle $(d=0.3 \mathrm{~mm})$. More details about fibers manufacturing are given in Ref 23 . All of the tested fibers have the diameter within the range $80 \pm 10 \mu \mathrm{m}$.

Fig. 2 The distribution and dispersion of MWCT in PA11_6 fibers 


\subsection{Methods}

The morphology of starting pellets and then fibers was analyzed using a scanning electron microscope (SEM) (SEM 3000, HR STEM S5500, Hitachi). In turn, the distribution and dispersion of MWCT in the fibers/starting pellets was analyzed using HR STEM S5500 in SEM and STEM mode. Samples for SEM and TEM observations were prepared using Leica UM6 positioned in a low-temperature chamber. Slides with a thickness of $80-90 \mathrm{~nm}$ were created using Diatome diamond knives which are suitable for trimming and sectioning. The process was performed using a temperature of $100{ }^{\circ} \mathrm{C}$ with the speed cutting set at $1 \mathrm{~mm} / \mathrm{s}$. The plain of cross sections was aligned with the direction of the extrusion process. The single fiber was cut parallel to its long axis. The SEM and TEM observations were made with a voltage of $30 \mathrm{kV}$. Additionally, the fiber surface was analyzed using SEM 5500.

The supermolecular structure, in terms of crystallinity and molecular orientation, was analyzed by using wide-angle x-ray scattering (WAXS) and differential scanning calorimetry (DSC). WAXS (D8 Discover, Bruker) measurements were performed using a diffractometer operated at the voltage of $40 \mathrm{kV}$ and a current of $40 \mathrm{~mA}$. $\mathrm{Cu} \mathrm{K} \alpha$ radiation (wavelength of $0.1542 \mathrm{~nm}$ ) was used. Measurements were performed in both transmission and scattering modes. Gebel geometry with a 0.6- mm slit and two Soller collimators applied on both sides were used in transmission mode. A highly sensitive 2D Vantec detector was used. The angular range of measurements $2 \theta$ was between $5^{\circ}$ and $30^{\circ}$ with a step of $0.01^{\circ}$ and with time of data accumulation at particular angular point of $0.2 \mathrm{~s}$. WAXS radial profiles were deconvoluted using the Pearson VII and Gauss function. The amount of the crystal, smectic, and amorphous phase of PA11 was determined by division of the area of crystal peaks $(001)$ and $(100)$ and $(010)+(110)$ by the total area (crystal, smectic and amorphous) scattered on PA11 from the radial profile after azimuthal averaging of intensity. Measurements were executed on well parallelised bundles of fibers. Firstly, background correction was performed. Thermal analysis was performed using DSC (Q-1000 (TA Instruments) placed in an aluminum hermetic pan in a nitrogen atmosphere. The utilized program was heat-cool-heat with a scan rate $10{ }^{\circ} \mathrm{C} /$ min. The crystallinity of all the materials was estimated using the standard equation:

$x_{\mathrm{c}}=\frac{\Delta H_{\mathrm{f}}}{\Delta H_{\mathrm{f}}^{0}}$,

where $x_{\mathrm{c}}$ is the crystallinity of measured sample, $\Delta H_{\mathrm{f}}$ is the heat of a sample normalized with respect to its mass, while $\Delta H_{\mathrm{f}}^{0}$ is the specific heat of fusion of $100 \%$ crystalline PA11
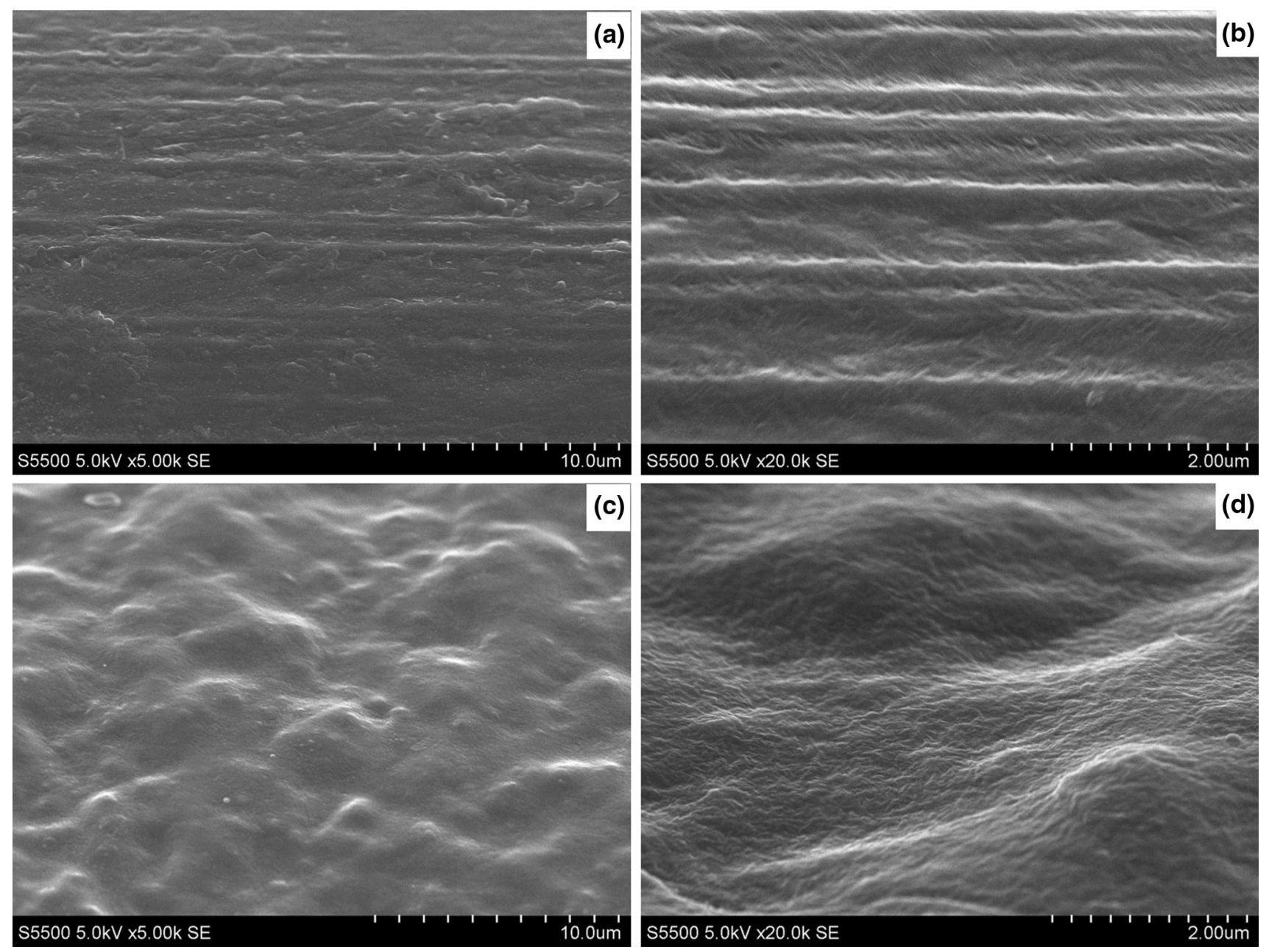

Fig. 3 Fibers surface: neat PA11 (a, b), PA11_6 (c, d) 
(a)

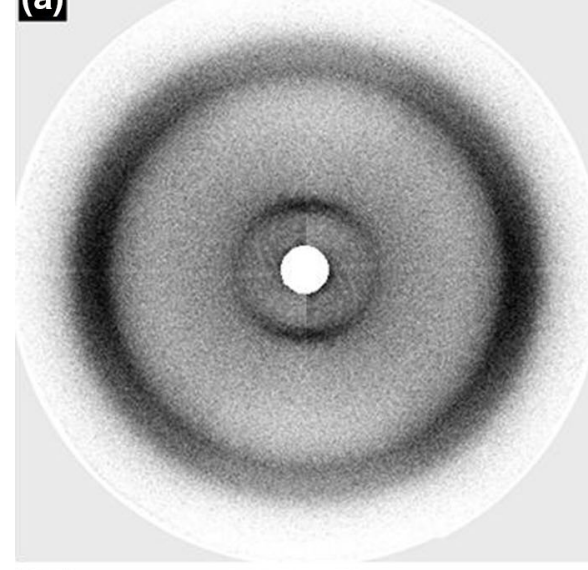

(c)

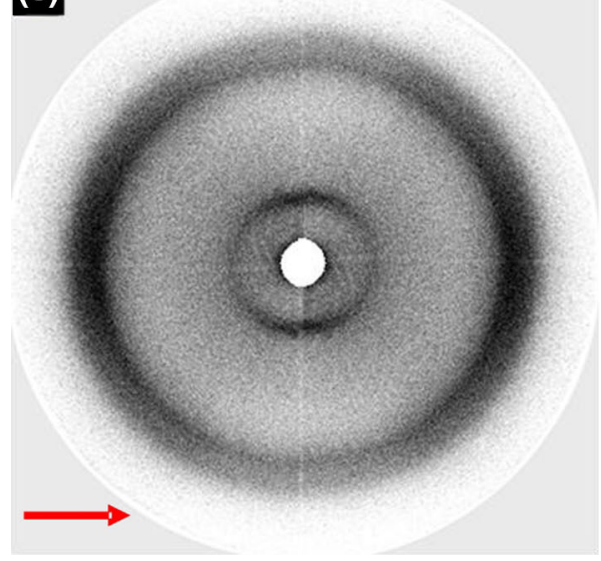

(b)

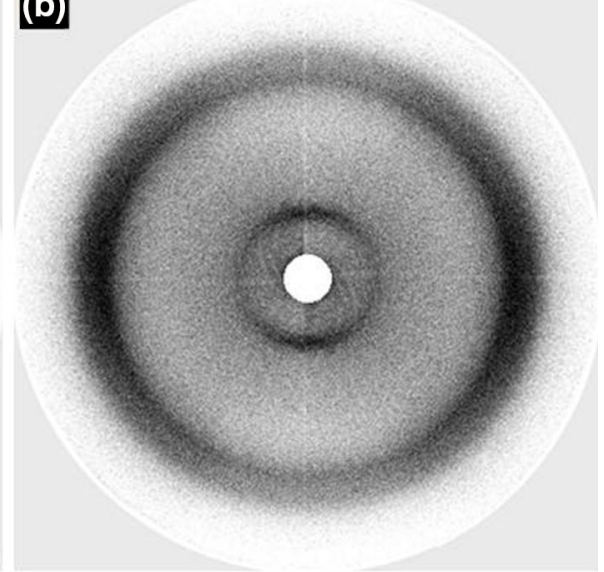

(d)

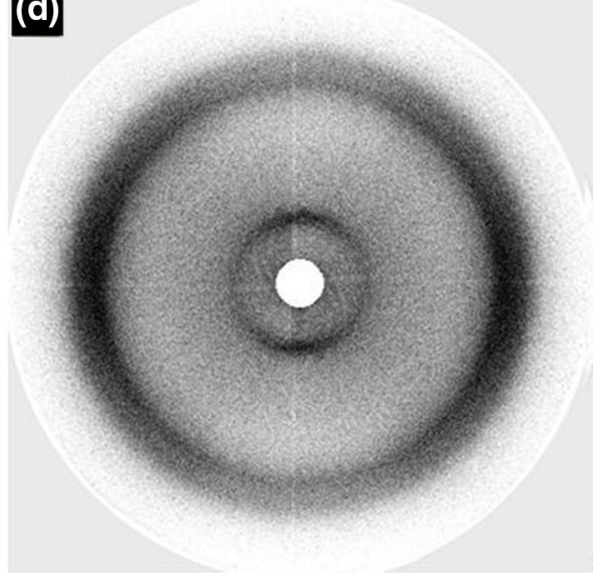

Fig. 4 WAXS patterns for fiber: PA11 (a), PA11_2 (b), PA11_4 (c), PA11_6 (d) (arrow indicates the direction of a fiber bundle)

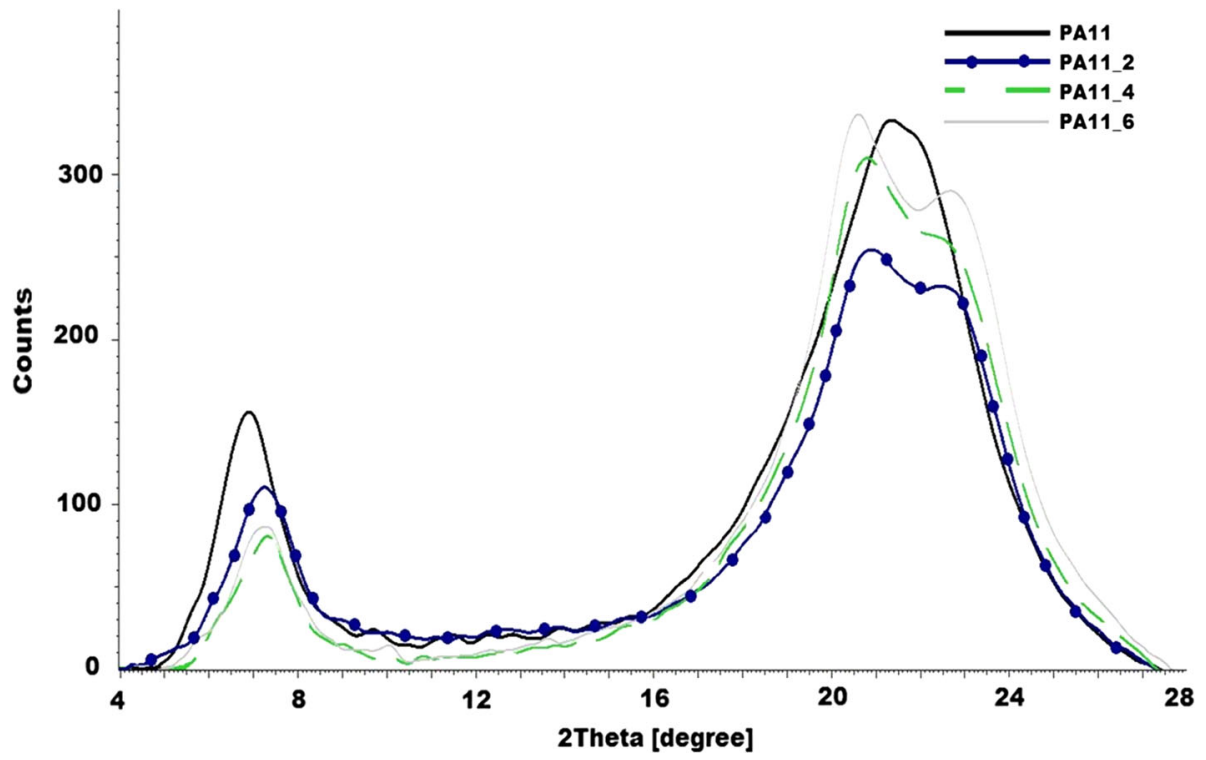

Fig. 5 Typical WAXS radial profiles for investigated PA11 and PA11/MWCT fiber

taken as $189.05 \mathrm{~J} / \mathrm{g}$ (Ref 5). For each composition, three measurements were conducted. The characteristic temperatures and calculated crystallinity for the pellets and fibers are presented in Table 2.
The mechanical properties of the extruded fibers were measured in tensile testing (MTS Tytron 250 instrument) according to the standard test method for tensile strength and Young's modulus of fibers (ASTM C1557-03(2013)). From one 
type of material, 10 single fibers were tested to failure at a constant cross-head displacement rate, with the same velocity equal to $10 \mathrm{~mm} / \mathrm{min}$. The used conditions were close to those in which the nanocomposite fibers will be worked. Then, the average values of tensile strength, elongation at break, and Young's modulus were calculated with taking the value of diameter into account.

\section{Results and Discussion}

\subsection{Morphology}

The state of dispersion in the one of starting pellets-PA11_6 is shown in Fig. 1. There are places with visible primary agglomerates with single nanotubes around them. Based on our experience with polymer/MWCT nanocomposites, these tubes are rather short when compared to other commercially available examples. Moreover, there are some visible black spots that indicate impurities in the material. It is highly probable that they are residues created by the carbon nanotubes manufacturing process (CVD-chemical vapor deposition) like carbon or the rest of the catalyst. Besides, the appearance of the MWCT used in this study as well as the state of dispersion is similar to that described by Mago et al. (Ref 19).

High shear forces and relatively long mixing time during extrusion process lead to the breakage of agglomerates, resulting in a satisfactory distribution and dispersion in the final fiber. The alignment of CNT is of great importance in achieving the desired mechanical properties of fibers. During extrusion, the movement of the melted polymer forces the orientation of the macromolecular chains. In turn, the orientation and alignment of carbon nanotubes is mostly triggered by the drawing or stretching of the extrudate (Ref 5, 7, 24]. TEM

Table 1 Interplanar distances, $d$ of angular positions $2 \theta$ of peaks for planar (001), (100), (010) + (110)

\begin{tabular}{lllc}
\hline & $\mathbf{( 0 0 1 )}$ & $\mathbf{( 1 0 0 )}$ & (010) + (110) \\
\hline Fiber & d, A & d, A & d, A \\
PA11 & 12,754 & 4088 & \\
PA11_2 & $12,035 \downarrow$ & $4200 \uparrow$ & $3898 \uparrow$ \\
PA11_4 & $12,052 \downarrow$ & $4237 \uparrow$ & $3862 \uparrow$ \\
PA11_6 & $11,926 \downarrow$ & $4237 \uparrow$ & $3862 \uparrow$ \\
\hline
\end{tabular}

images taken from thin sections prepared along the PA11/ MWCT fiber are shown in Fig. 2. The red arrows show the direction of the extrusion and drawing. It can be seen that there is no alignment of MWCT in the extrusion direction. This is contrary to, for instance, polycarbonate/MWCT fibers (Ref 24) or those reviewed in Ref 5-7 prepared by melt spinning. The reason for this could be related to a too low drawing velocity that was described for polyethylene/MWCT (Ref 25) and polycarbonate/MWCT fibers (Ref 26). On the other hand, the dimensions of the used MWCTs can be a factor affecting the lack of orientation of tubes within the fibers what was also presented by Pötschke et al. in Ref 27). It is highly probable that very short MWCTs move in the polymer much easier than longer MWCT. Hence, during the crystallization step of fibers (after extrusion), the MWCT can lose the arrangement.

Furthermore, the qualitative analysis of PA11/MWCT fiber was conducted. Figure 3 presents the surface of the extruded fibers using SEM. Neat PA11 fiber (Fig. 3a, b) is characterized by grooves aligned parallel to the main axis, as a result of the fabrication process. There is visible surface continuity and it can be stated that polymer's chains are oriented in the drawing direction. In the case, PA11_6 fiber's topography is altered significantly (Fig. 3c, d). It can be seen that the surface is not as smooth like it is for neat PA11 fibers. Moreover, there are some defects on the surface including cracks and knobs. Such discontinuity of the surface can diminish the mechanical properties (Ref 4-7). Most likely, the presence of MWCTs makes the extrusion process much more difficult, resulting in macroscopic defects of the fiber.

All of the predictions made about orientation of polymer chains and MWCT in the fibers using the microscopic images will be discussed and compared in the sections 3.2 and 3.3 by WAXS and DSC analysis, respectively.

\subsection{Molecular Structure}

The supermolecular structure was analyzed using WAXS and DSC. Figure 4 illustrates typical WAXS patterns registered for various fibers (A: PA11; B: PA11_2; C: PA11_4; D: PA11_6). All of the fiber samples indicate the molecular orientation of PA11 crystal phase. The radial profile of pure PA11 fiber shows several peaks attributed to triclinic crystal structure: crystal phase $\delta^{\prime}(001)$ at $2 \theta=4^{\circ}$ and (100) at $2 \theta=21^{\circ}$; smectic $\gamma$ phase at $2 \theta=20.5^{\circ}$; amorphous phase at $2 \theta=10^{\circ}$ and $2 \theta=19.5^{\circ}$ (Fig. 5). The radial profile of the PA11 nanocomposite fiber shows additional peaks that can be attributed to $(010)+(110)$ at $23.5^{\circ}$ and a weakly visible peak at
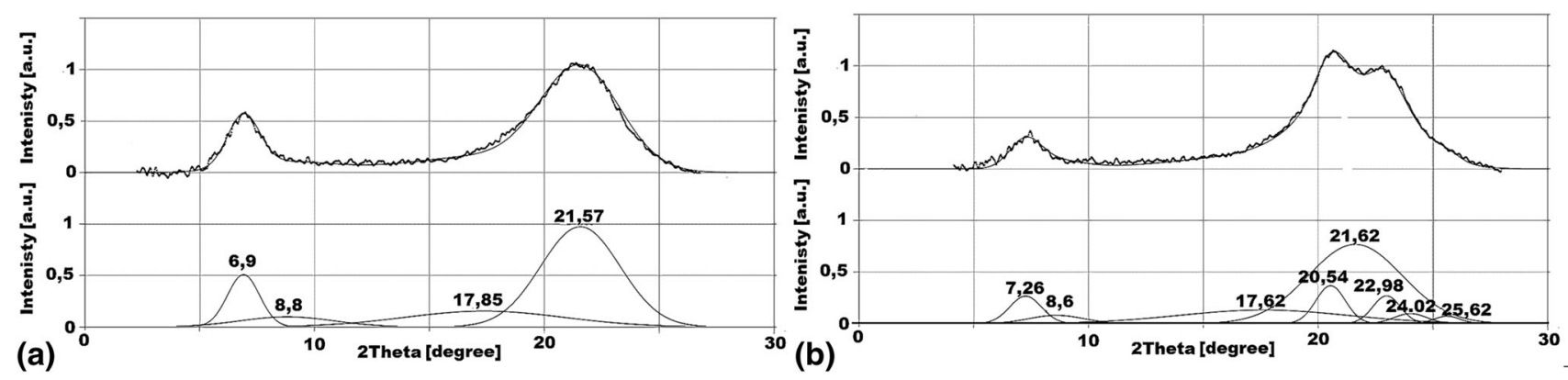

Fig. 6 Examples of fittings of WAXS radial profiles PA11 (a), PA11_6 (b) 
about $2 \theta=26^{\circ}$ corresponding to MWCT presence (Fig. 5). The radial profile of pure PA11 is characteristic for polyamides formed after having been quenched in an ice bath. Peaks at $(100)$ at $2 \theta=21^{\circ},(010)+(110)$ at $23.5^{\circ}$ are characteristic for polyamides crystallizing in $\alpha$ form during isothermal crystallization at high temperature ( $\operatorname{Ref} 10,28,29)$. It is evident that the addition of MWCT promotes $\alpha$ phase formation. This is probably due to the thermal capacity of nanotubes or the crystallization in temperatures higher than $95^{\circ} \mathrm{C}$. This effect was defined already for the PA11/MWCT pellets, which were then used for fiber formation. There is a small shift of PA11 (001) and (100) reflections observed for PA11/MWCT in comparison with pure PA11 (Fig. 5). Those shifts correspond to a small decrease in d crystal spacing connected to the $c$ axis (001) of the lattice cell diameter and an increase of a (100) in the presence of MWCT. In turn, the position of $(010)+(110)$ in the case of PA11/MWCT fibers does not change significantly (Table 1). Slichter et al. observed a change of phase from $\delta^{\prime}$ to $\alpha$ under pressure as a reduction in spacing on the (001) planes by compression of the hydrogen bond between macromolecules in plane $(010)$ by high pressure. Reduction of $\mathrm{N}-\mathrm{H} \cdots \mathrm{O}$ distance influences a change in the angle $\beta$. Quite similar effects were observed in the samples that were reported already (Ref 12,13).

Examples of the fittings of WAXS radial profiles of the investigated fibers using the Pearson VII (crystal phase) and Gauss (smectic and amorphous phase) function are shown in Fig. 6. It can be seen from Fig. 7 that a relatively small addition of MWCT leads to an increase of PA11 crystalline and smectic phase content. This is because of the effect of additional

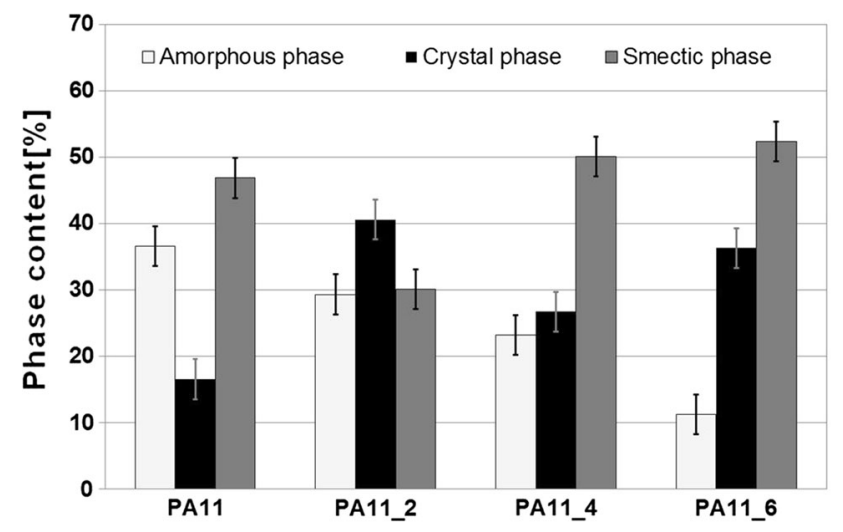

Fig. 7 Phases content for pure PA11 and PA11/MWCT at room temperature nucleation on MWCT and/or more effective drawing in the presence of MWCT.

\subsection{Thermal Properties}

A thermal analysis was conducted for the pellets and fibers, and the results are collected in Table 2. The addition of MWCT has a visible effect on the temperature of crystallization $\left(T_{\mathrm{c}}\right)$ which is $8{ }^{\circ} \mathrm{C}$ higher for PA11 fibers with 6 wt.\% MWCT than for neat PA11. This confirms the nucleation effect of MWCT on PA11 in the fibers as well as in the pellets (Table 2). The addition of MWCT has a much smaller influence on the melting point $\left(T_{\mathrm{m}}\right)$ as well as the crystalline content of PA11 fibers. DSC results show that the crystals melt in the wide temperature range between 150 and $170{ }^{\circ} \mathrm{C}$ (Fig. 8). Interestingly, there is a drop in melting point for first and second heating equal to $3{ }^{\circ} \mathrm{C}$ because of the MWCT presence. Such phenomenon was observed by Huang et al. for PA11 doped with functionalized MWCT (Ref 20) and could be caused by different types of crystals. If smaller, imperfect/defected crystals are formed, they have a lower melting temperature. It is worth noticing the typical tiny peak just before the main melting range on the neat PA11 curve is associated with the occurrence of polymorphism in PA11 (Ref 14). The degree of crystallinity was determined using Eq 1. According to Table 2, there is not a clear dependence of PA11 crystalline in MWCT function for pellets and fibers. Generally, the crystalline content decreases for filled PA11 but there are some deviations, especially for fibers. Nevertheless, these differences in crystallinity are in agreement with that observed in the WAXS patterns.

\subsection{Mechanical Properties}

It was shown that PA11/MWCT fibers possess unsatisfactory orientation of the macromolecules in the direction of drawing. Besides, the MWCT alignment is not as high as it could be because the drawing conditions are not optimized. Nonetheless, we would like to show whether or not fully ordered MWCTs affect the mechanical performance of PA11 fibers. Figure 9(a)-(c) presents the Young's modulus, tensile strength, and elongation at break of the PA11/MWCT fibers. It is known, that at low strains up to the yield point, the MWCTdoped PA11 fibers show higher stress (Ref 5, 7, 30). It is expressed by an increase in Young's modulus clearly visible in Fig. 9(a). Unfortunately, the obtained values are lower than expected. For PA11_6 fibers, the Young's modulus is almost $600 \mathrm{MPa}$. Compared to literature, the lowest found values of

Table 2 Thermal properties for pellets and fibers of PA11 and MWCT-doped PA11

\begin{tabular}{|c|c|c|c|c|c|c|c|c|c|c|}
\hline \multirow{2}{*}{ Form } & \multirow{2}{*}{ Material } & \multicolumn{5}{|c|}{ First heat } & \multicolumn{3}{|c|}{ Second heat } & \multirow{2}{*}{$\begin{array}{c}\text { Cooling } \\
T_{\mathrm{c}},{ }^{\circ} \mathrm{C}\end{array}$} \\
\hline & & \multicolumn{2}{|c|}{$T_{\mathrm{g}},{ }^{\circ} \mathrm{C}$} & $T_{\mathrm{m}},{ }^{\circ} \mathrm{C}$ & $\Delta \boldsymbol{H},{ }^{\circ} \mathrm{CJ} / \mathrm{g}$ & $X_{\mathrm{c}}, \%$ & $T_{\mathrm{m}},{ }^{\circ} \mathrm{C}$ & $\Delta \boldsymbol{H}, \mathbf{J} / \mathbf{g}$ & $X_{\mathrm{c}}, \%$ & \\
\hline \multirow[t]{4}{*}{ Pellets } & PA11 & 45.89 & 101.2 & 189.3 & 51.47 & 27.22 & 188.7 & 46.74 & 24.72 & 163.1 \\
\hline & PA11_2 & 45.88 & 104.4 & 188.4 & 58.42 & 30.91 & 188.4 & 52.96 & 28.01 & 173.8 \\
\hline & PA11_4 & 47.47 & 85.01 & 186.6 & 43.23 & 22.87 & 186.7 & 40.44 & 21.39 & 171.9 \\
\hline & PA11_6 & 43.86 & 80.02 & 185.2 & 39.27 & 20.77 & 185.2 & 47.42 & 25.08 & 171.5 \\
\hline \multirow[t]{4}{*}{ Fibers } & PA1 $\overline{1}$ & 58.54 (Peak) & - & 186.9 & 40.05 & 21.18 & 1878.0 & 69.59 & 36.81 & 162.9 \\
\hline & PA11_2 & 53.50 & - & 186.4 & 39.54 & 20.92 & 185.8 & 61.45 & 32.51 & 173.2 \\
\hline & PA11_4 & 47.91 & - & 185.7 & 52.24 & 27.63 & 184.5 & 36.78 & 19.45 & 171.3 \\
\hline & PA11_6 & 51.42 & - & 183.5 & 36.49 & 19.30 & 184.3 & 42.91 & 22.69 & 170.4 \\
\hline
\end{tabular}




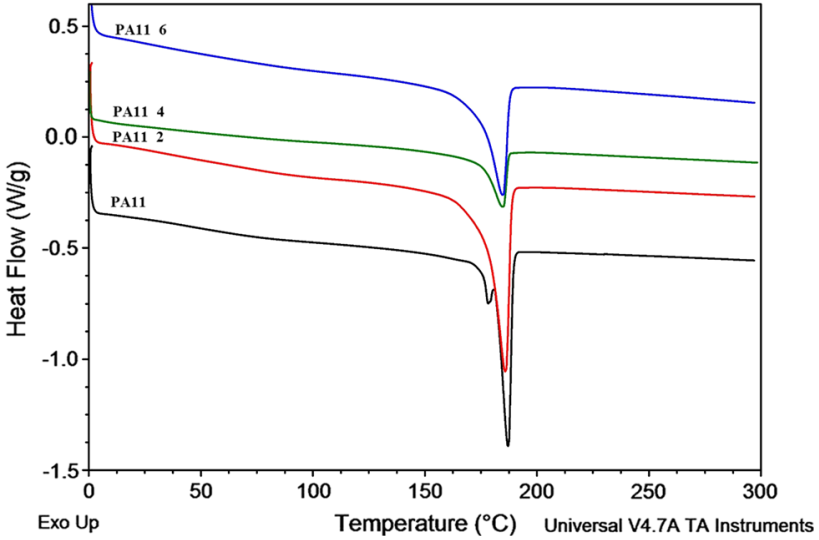

Fig. 8 Melting peak for unfilled and filled PA11and PA11/MWCT fibers. Thermograms are recorded during the 2 nd heating

Young's modulus for others polymer/MWCT fibers are at the level of $500 \mathrm{MPa}$. In turn, the elongation at break for more stiff fibers, here 4 and 6 wt.\% MWCT, is decreased. This is shown in Fig. 9(c). For industrial application the most important factor is tensile strength, which is reported as to be between 0.1 and $7 \mathrm{GPa}$ (Ref 5, 7, 30). Generally, the tensile properties of fibers are dependent on (i) orientation of macromolecules chains, (ii) dispersion of the MWCT, and (iii) alignment of the MWCT. These factors are expressed by crystalline content. Following the calculated percentage of $X_{\mathrm{c}}$, it should be assumed that the MWCT-doped PA11 fibers should have lower tensile strength. This is because the amount of crystalline phase in fibers is lower when MWCTs are added. Following the results in Table 2, there are a few deviations in $X_{\mathrm{c}}$ values, which make a comparison between tensile and crystalline content difficult. Therefore, we presume that there are other factors affecting a significant reduction of PA11/MWCT strength. The first one is related to the described MWCT agglomerates in the fibers and the mentioned impurities occur in the material (see Fig. 2). Weakly dispersed MWCT bundles can act as stress concentration points what was also noticed by Pötschke et al. (Ref 24). The second reason is associated with the nature of the used MWCT. On one hand, the presence of impurities can work as an intrinsic crack. On the other hand, MWCTs with too short of a length (less than $1.8 \mathrm{~nm}$ ) are not able to connect the PA 11 chains together, resulting in easy gliding of macromolecular chains. Finally, the third reason for decreasing tensile strength of the PA11 fibers after MWCT addition is linked to the macroscopic effects. According to microscopic investigation in section 3.1, the surface of the fiber after MWCT addition possesses visible defects and a much rougher face. Contrary to the neat PA 11 fibers that have a surface which is flat and smooth (see Fig. 3). Hence, even small discontinuities like knobs or oval grooves, which are easily noticeable on the PA/ MWCT surface, can act as micronotches during tensile strength. It is highly probable that macroscopic effects have stronger influence of mechanical strength of PA11/MWCT fibers.

\section{Conclusions}

In the present work, the nanocomposite fibers made of Polyamide 11 and 2, 4, and 6 wt.\% of MWCT (PA11/MWCT) were characterized by their molecular structure, microstructure, and mechanical properties. WAXS analysis shows that fibers
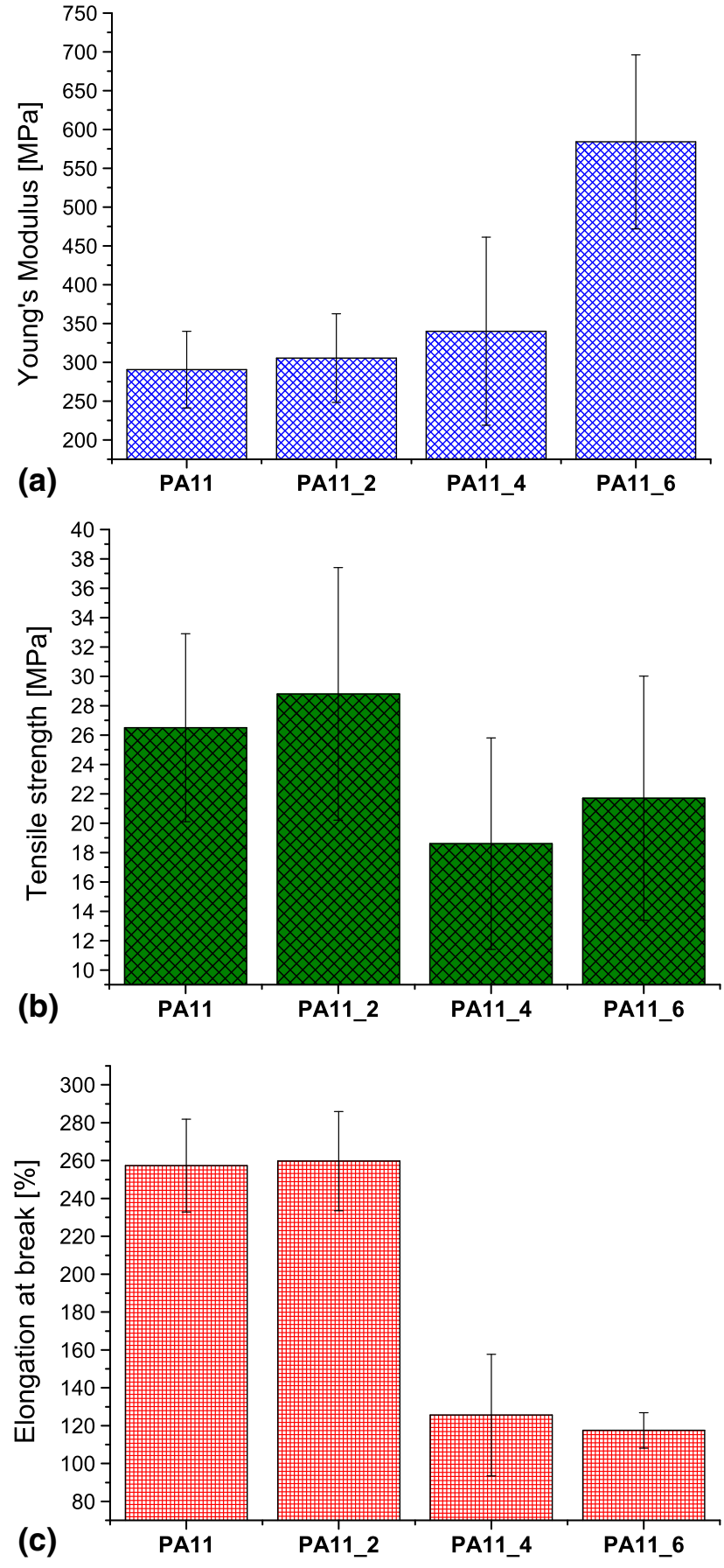

Fig. 9 Mechanical properties of nanocomposite fibers: Young's modulus (a), tensile strength (b), elongation at brake (c)

achieve the molecular orientation of PA11 crystal phase but the addition of MWCT leads to a reduction of expected orientation in these fibers. These results were confirmed by microscopic investigations where MWCTs occur in the form of agglomerates and they are not aligned in the drawing direction. Deep analysis of PA11 polymorphism suggests that MWCTs pronounce the creation of a stable $\alpha$ phase in both the starting pellets and fibers. Besides, a third phase (smectic) in the PA11/ MWCT fibers was found. Crystallization temperature increases after MWCT addition. This confirms that MWCT works as a nucleating factor for PA11. Contrary to a decreased melting 
range for the filled PA11 fibers, this is related to the formation of defected crystals. Generally, the degree of crystalline is diminished when MWCTs are present, but some visible deviations occur. Mechanical testing shows that just Young's modulus increases after MWCT addition. More stiff fibers have lower elongation at break. In turn, the changes in tensile strength of fibers are related to various parameters. There is a lack of fiber orientation, MWCT agglomerations, and surface defects of the fiber. All of these factors cause the significant reduction of their strength. In order to achieve good quality PA11/MWCT fibers, it is necessary to apply higher drawing of extrudate which will lead to improved strength. Merely by improving mechanical behavior, these fibers can be used in many industrial sectors, mainly in as a precursor for smart, conductive fabrics in textile world.

\section{Open Access}

This article is distributed under the terms of the Creative Commons Attribution 4.0 International License (http:// creativecommons.org/licenses/by/4.0/), which permits unrestricted use, distribution, and reproduction in any medium, provided you give appropriate credit to the original author(s) and the source, provide a link to the Creative Commons license, and indicate if changes were made.

\section{References}

1. J.A. Brydson, Polyamides and Polyimides in Plastics Mater, 7th ed., Elsevier, London, 1999, p 478-530ISBN 978-0-7506-4132-6

2. S. Eichhorn, J.W.S. Hearle, M. Jaffe, and T. Kikutani, Handbook of Textile Fiber Structure, Fundamentals and Manufactured Polymer Fibres, Woodhead Publishing. ISBN: 978-1-84-569-650-4, 2009

3. ARKEMA data sheets, Rilsan PA11: created from a renewable source

4. B.G. Min, H.G. Chae, M.L. Minus, and S. Kumar, Polymer/Carbon Nanotube Composite Fibers-An Overview, Res. Signpost Transw. Res. Netw., 2009, 2, p 43-73

5. Y. Zhang, J. Meng, E.C. Green, N. Tojaddod, H. Li, and M.L. Minus, Structural Polymer-Based Carbon Nanotube Composite Fibers: Understanding the Processing-Structure-Performance Relationship, Mater, 2013, 6(6), p 2543-2577

6. Y. Liu and S. Kumar, Polymer/Carbon Nanotubes Nano Composite Fibers-A Review, Appl. Mat. Interf., 2014, 6(9), p 6069-6087

7. H. Deng, Q. Fu, R.R. China, E. Bilotti, T. Peijs, The Use of PolymerCarbon Nanotube Composites In Fibers-Chapter 21 in PolymerCarbon Nanotube Composites: Preparation, Properties and Applications, T. McNally, P. Pötschke, Ed., Woodhead Publishing, ISBN 978$1-84569-761-7,2011$

8. S. Chatterjee, F.A. Nuesch, and B.T.T. Chu, Crystalline and Tensile Properties of Carbon Nanotube and Graphene Reinforced Polyamide 12 Fibers, Chem. Phys. Lett., 2013, 557, p 92-96

9. L. Jolly, A. Tidu, J.J. Heizmann, and B. Bolle, Microstructure Evolution in Polyamide PA11 Under Small Uniaxial Extension, Polymer, 2002, 43(25), p 6839-6851

10. Q. Zhang, Z. Mo, H. Zhang, S. Liu, and S.Z.D. Cheng, Crystal Transitions of Nylon 11 Under Drawing and Annealing, Polymer, 2011, 42(13), p 5543-5547
11. W.P. Slichter, Crystal Structures in Polyamides Made from $\omega$-Amino Acids, J. Polym. Sci., 1959, 36(130), p 259-266

12. B.A. Newman, T.P. Sham, and K.D.A. Pae, High-Pressure X-ray Study of Nylon 11, J. Appl. Phys., 1977, 48(10), p 4092-4098

13. P. Ricou, E. Pinel, and N. Juhasz, Temperature Experiments for Improved Accuracy in the Calculation of Polyamide-11 Crystallinity by X-ray Diffraction, Adv. X-ray Anal. Int. Cent. Diffr. Data, 2005, 48, p $170-175$

14. A. Kawaguchi, T. Ikawa, Y. Fujiwara, M. Tabuchi, and K. Monobe, Polymorphism in Lamellar Single Crystals of Nylon 11, J. Macrom. Sci. Phys., 1981, 20(1), p 1-20

15. P.K. Chen, B.A. Newman, J.I. Scheinbeim, and K.D. Pae, HighPressure Melting and Crystallization of Nylon 11, J. Mater. Sci., 1985, 20, p 1753-1762

16. H.H. Yu, Crystal Phase Transformations in Nylon 11, Mater. Chem. Phys., 1998, 56, p 289-293

17. S.S. Nair, C. Ramesh, and K. Tashiro, Crystalline Phases in Nylon-11: Studies Using HTWAXS and HTFTIR, Macromolecules, 2006, 39, p 2841-2848

18. A. Mollova, R. Androsch, D. Mileva, Ch Schick, and A. Benhamid, Effect of Supercooling on Crystallization of Polyamide 11, Macromolecules, 2013, 46, p 828-835

19. G. Mago, D.M. Kalyon, and F.T. Fisher, Nanocomposites of Polyamide-11 and Carbon Nanostructures: Development of Microstructure and Ultimate Properties Following Solution Processing, J. Polym. Sci. B, 2011, 49, p 1311-1321

20. H.S. Wang, M. Liu, T. Zhang, W.D. Tjiu, W.Ch. He, and X. Ch.Lu, Morphology, Thermal, and Rheological Behavior of Nylon 11/MultiWalled Carbon Nanotube Nanocomposites Prepared by Melt Compounding, Polym. Eng. Sci., 2009, 49, p 1063-1068

21. S. Lao, M.F. Kan, C.K. Lam, D.Z. Chen, J.H. Koo, T. Moon, M. Londa, T. Takatsuka, E. Kuramoto, G. Wissler, L. Pilato, Z.P. Luo, Polyamide 11-Carbon Nanotubes Nanocomposites: Processing, Morphological and Property Characterization, Conference Proceedings SAMPE, Seattle, WA, May 2010, p 17-20

22. D. Carponcin, E. Dantras, G. Aridon, L. Levallois, L. Cadiergues, and C. Lacabanne, Evolution of Dispersion of Carbon Nanotubes in Polyamide 11 Matrix Composites as Determined by DC Conductivity, Compos. Sci. Technol., 2012, 72, p 515-520

23. P. Latko, R. Kozera, A. Salinier, and A. Boczkowska, Non-Woven Veils Manufactured from Polyamides Doped with Carbon Nanotubes, Fibr. Text. East. Eur, 2013, 21(6(102)), p 45-49

24. P. Pötschke, H. Brunig, A. Janke, D. Fischer, and D. Jehnichen, Orientation of Multiwalled Carbon Nanotubes in Composites with Polycarbonate by Melt Spinning, Polymer, 2005, 46, p 1035510363

25. A.B. Sulong, J. Park, Ch.H. Azhari, and K. Jusoff, Process Optimization of Melt Spinning and Mechanical Strength Enhancement of Functionalized Multiwalled Carbon Nanotubes Reinforcing Polyethylene Fibers, Compos. B, 2011, 42, p 11-17

26. M. Sennett, E. Welsh, J.B. Wright, W.Z. Li, and Z.F. Ren, Dispersion and Alignment of Carbon Nanotubes in Polycarbonate, Appl. Phys. A, 2003, 76, p 111-113

27. P. Pötschke, A.R. Bhattacharyya, and A. Janke, Melt Mixing of Polycarbonate with Multiwalled Carbon Nanotubes: Microscopic Studies on the State of Dispersion, Eur. Pol. J., 2004, 40, p 137-148

28. J.I. Scheinbeim, J.W. Lee, and B.A. Newman, Ferroelectric Polarization Mechanisms in Nylon 11, Macromolecules, 1992, 25(14), p 37293732

29. S. Dasgupta, W.B. Hammond, and W.A. Goddard, Crystal Structures and Properties of Nylon Polymers from Theory, J. Am. Chem. Soc., 1996, 118, p 12291-12301

30. W. Chen, X. Tao, and Y. Liu, Carbon Nanotube-Reinforced Polyurethane Composite Fibers, Compos. Sci. Technol., 2006, 66, p 3029-3034 